\title{
Cartesianization of Complex Forms in Saidou' Spaces
}

\author{
Nourddin Saidou \\ Euromed University 32 Rue Meknes, Morocco \\ n. saidou@insaueuromed. org
}

\begin{abstract}
In this work, we propose to define a new space that will be called locally Cartesian space (Saidou'Spaces). It is a space defined by a new topology whose fundamental system of neighborhood are compact cartesian subsets, theses subsets were already defined during our previous work, "Geometrical introduction to Cartesian geometry in complex shapes see [15]. It will have the same properties of a Banach space. Then, we will give the steps of a cartesianization method of complex shapes or region in this space. The objective sought by this technique is to find a cartesian form (denoted Car(A)), that approximates any form A, see Figure1 below. It should be noted that cartesianization will be a generalization of the polygonization whish remains the object of research for several researchers in the spaces $R^{2} \operatorname{Ref}[12]$ [13] [6].
\end{abstract}

Keywords: Saidou' Spaces, Banach space, regular Cartesian.

2010 MSC No: 46A16, 32-XX
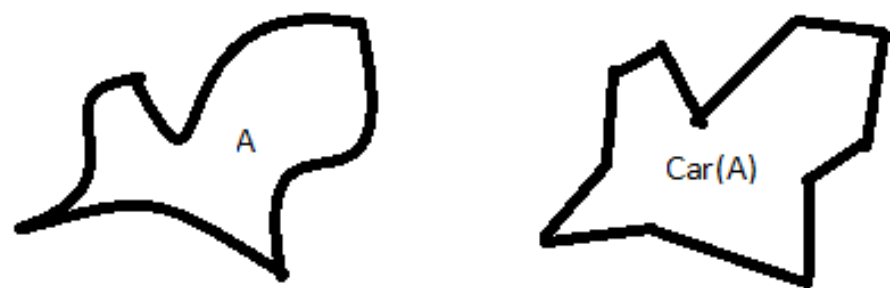

Figure(1): The complex region in the left and its cartesian profile in the right

\section{Introduction}

As already explained in our previous work see saidou [15], to study a cartesian form (Polygonal in $R, R^{2}, R^{3}$ or polytopes in $R^{n}$ ) is easier to study than any form. To say easy to study is to say easy to control and manage, because the borders are composed of linear parts, see Figure 1 above. It should be noted that the Cartesian subsets are a finite union of polytopes in infinite dimensional spaces, see Ref [7] [16] [2]. The polytopes are always convex closed bounded but the Cartesian subsets are closed bounded and not necessarily convex. The main objective of this work is to build a mathematical algorithm in order to find an approximate cartesians profile to any complex shapes. This approximation will depend on the number of extremal or exposed points chosen. See Figure 2 below. To do this, we introduce the new locally cartesian space as a workspace that also possesses the properties of a Banach space. Thus, we could use already studied notions of geometric analysis of Banach spaces, namely hyperplanes, the convex hull, the extremal and 
exposed points of a convex subsets see Ref [1][11][16].
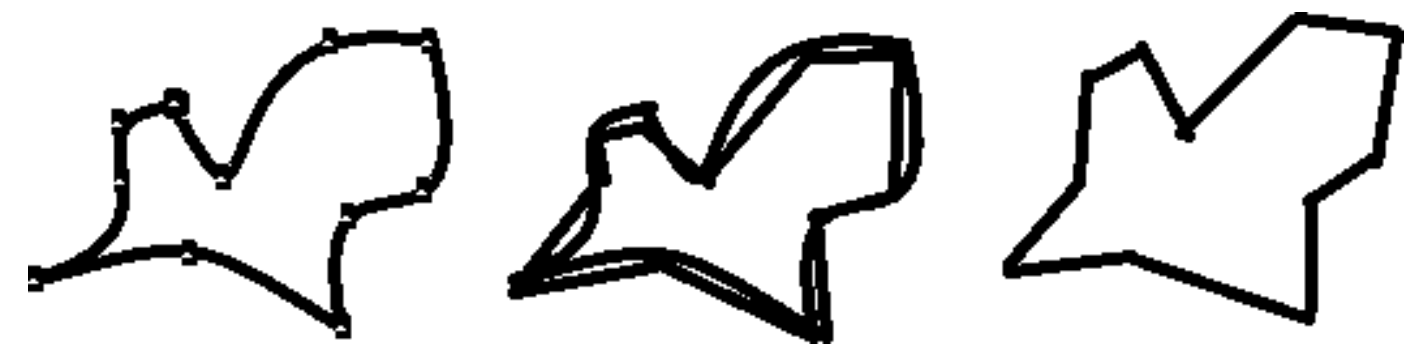

Figure(2): The complex region with some exposed points in the left and the cartesianization following exposed points in the middle, its cartesian profile in the right.

\section{Definitions and Preliminaries}

Le $E$ be a Banach space, $E^{\prime}$ its dual and <. , . > the dual product.

1. $H$ is called an hyperplan in $\mathrm{E}$ if and only if, there exists a linear form $x^{\prime}$ belonging to the dual of E such that: $H=\{y \in E,\langle x, y\rangle=\alpha, \alpha \in R\}$.

2. $D=\left\{\mathrm{y} \in \mathrm{E},\left\langle\mathrm{x}^{\prime}, \mathrm{y}>\leq \quad \alpha.\right\}\right.$ is called the lower half space defined by $x^{\prime}=\alpha$.

3. $D=\left\{y \in E,<x^{\prime}, y>\geq \alpha\right\}$. is called the upper half space defined by $x^{\prime}=\alpha$.

4. A set $\mathrm{C}$ in $\mathrm{E}$ is said to be convex if and only if, for each $\mathrm{x}, \mathrm{y}$ in $\mathrm{C}$, the segment $[\mathrm{x}, \mathrm{y}]$ is included in $\mathrm{C}$.

5. Let $A$ a subset in E. $C o(A)=\left\{y=\sum \alpha_{i} x_{i}, \sum \alpha_{i}=1, x_{i} \in A\right\}$ is said the convex of $A$. see Figure 3 below. It is the convex combination of the elements of $\mathrm{A}$. We will also say that it is the convexified set of $A$.

The convexification is to find the convexified set of $A$.
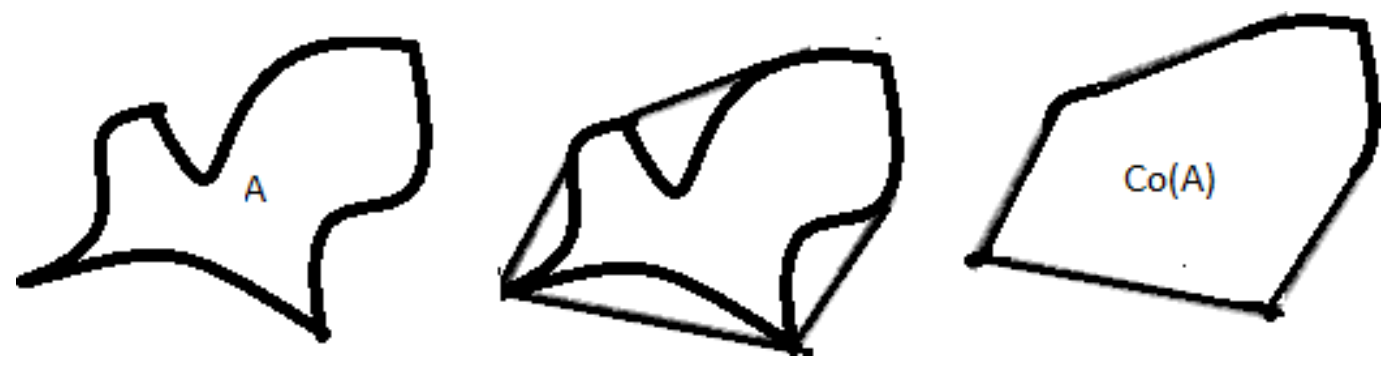

Figure(3): The subset $A$ in the left, its convexification in the middle, its convex hull in the right

6. A point $\mathrm{e}$ is said to be an extremal point of a closed subset $\mathrm{C}$ iff there is not any $\mathrm{x}, \mathrm{y}$ in $\mathrm{C}$ such that $\mathrm{e}$ is in $[\mathrm{x}, \mathrm{y}]$ which is means that: $e=\alpha x+(1-\alpha) y$ implies that $e=x=y$. see Figure 4 below.

7. convex closed subset $\mathrm{C}$ iff there exists a $x^{\prime} \in E^{\prime}$ such that: $\langle x, e\rangle=\sup _{y \in C}\left\langle x^{\prime}, y\right\rangle$ and $<x, e>><x, y>\forall y \# e$. See Figure 4 below. 


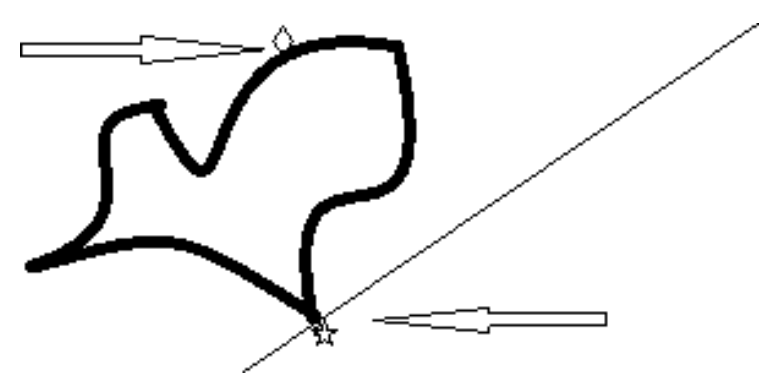

Figure(4): The extremal point above of A, and an exposed point of A with a support hyperplan below.

Remark 2.1 It is easy to prove that H, D are closed convex sets in E.

Definition 2.2 . C is said to be a regular Cartesian set of $\mathrm{E}$ if and only if, $\mathrm{C}$ is the intersection of a finite family $I$ of $D_{i}$, with $D_{i}$ are half-spaces of $E$.

$$
\boldsymbol{C}=\bigcap_{i \in I}\left(D_{i}\right)
$$

The regular cartesians subsets are the same of polytopes or simplexes sets in $R^{n}$.

\section{Example 2.3.}

- A square is a regular Cartesian set defined by the intersection of four half spaces.

$[0,1] \times[0,1]$ is the intersection of the following four half-spaces:

The half space below the line $y=1$, the half space above $y=0$, the half space to the left of the vertical axis $\mathrm{x}=1$ and the half space to the right of the vertical axis $\mathrm{x}=0$.

- A triangle is a regular Cartesian set defined by the intersection of three half- spaces. see Figure 5 above.

Now, we have all the necessary assets to pronounce the first definition of a cartesian set. In fact, it would be a concatenation of regular Cartesian sets.

Definition 2.4 Let $\mathrm{C}$ be a set of $\mathrm{E}$. We say that $\mathrm{C}$ is Cartesian if and only if $\mathrm{C}$ is a finite union of regular Cartesian sets connected to each other.

$$
\mathbf{C}=U \mathbf{j} \in \mathbf{J} \cap \mathbf{i} \in \mathbf{I}(\mathbf{D i}, \mathbf{j})
$$

I, J are finites. See Figure 6.
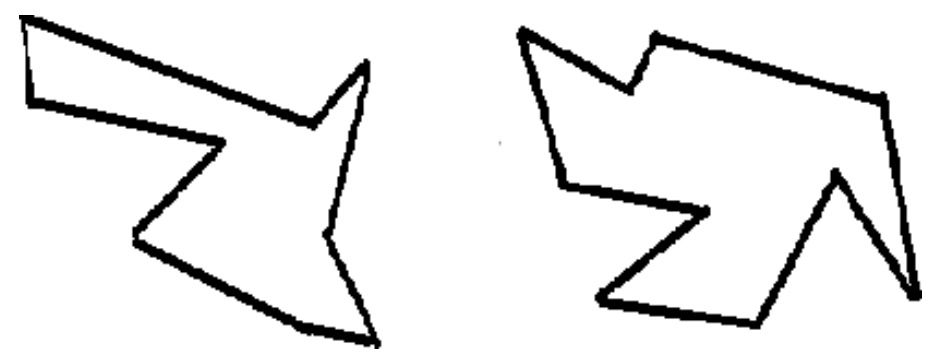

Figure(6): Cartesians sets

Now, after to have defined the cartesian sets, we can give the definition of locally cartesian spaces called Saidou'Spaces. 


\section{Definition of localy cartesians space ( Saidou' Spaces)}

Definition 3.1 Let $E$ a Banach spaces, $E$ is said to be a loclay cartesian space ( Named Saidou'Spaces), if and only if, it has a fundamental system of compact cartesians neighbourds which is means that each $\mathrm{x}$ in $\mathrm{E}$ has a compact cartesian neighbourd.

Remark 3.2 Since the union of cartesian sets is cartesian and the intersection of cartesian sets is cartesian and the empty set is cartesian, we can say that the family of cartesian sets of E consititue a topology which will be called the cartesian topology.

\section{Example 3.3 .}

1. A cartesian space is a discret space, because for a finte set of points, we can construct a cartesian set.

2. A space $\left(\mathrm{E}, \mathrm{II} . \mathrm{II}_{1}\right)$ with the norm 1 is a Saidou' S pace, because its boule $\left(\mathrm{B}(0,1), \mathrm{II}\right.$. $\left.\mathrm{II}_{1}\right)$ is cartesian.

The aim of this paper is not to study the topological property and gives results that characterize the saidou' spaces, but it is to expalin the algorithm of cartesianization of complex forms.

\section{Definitions of Convexification by parts}

Let $\mathrm{E}$ a localy cartesian space.

Let $A$ a compact subset of $\mathrm{E}$. In this section we will explain the convexification by parts of $A$, as you will remark it is different from the convexification of $A$, because this technique allows us to have a set that contains the set $A$ and smaller than the convex hull $C o(A)$ of $A$. The convex hull by part of $A$ will denoted by $\operatorname{Cop}(A)$.

How to construct $\operatorname{Cop}(A)$ ?

In the first step, we partition $A$ as an union of compact convex subsets and compact non convex subsets.

$$
A=\left(C_{1} \cup C_{2} \cup \ldots \cup C_{n}\right) \cup\left(D_{1} \cup \ldots \cup D_{m}\right)
$$

With $C_{i}$ are compact convex subsets of $A$ and $D_{j}$ are compact non convex subsets of $A$.

We illustrate with an example of $1 \leq i \leq 2$ and $1 \leq j \leq 2$ see Figure 7 below.
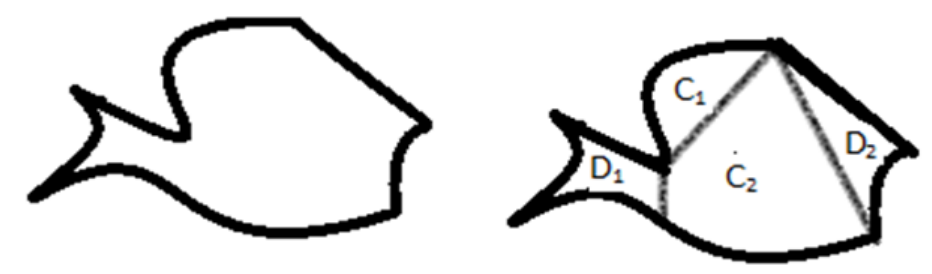

Figure(7): The subset $A$ in the left and its partitioning $A=\left(C_{1} \cup C_{2}\right) \cup$ $\left(D_{1} \cup D_{2}\right)$ in the right.

Then, the second step is to convexifie each $D_{j} \quad$ i.e.d to consider $\operatorname{Co}\left(D_{j}\right)$.

Then we put.

$$
\operatorname{Cop}(A)=\left(C_{1} \cup C_{2} \cup \ldots \cup \cup C_{n}\right) \cup\left(\operatorname{Co}\left(D_{1}\right) \cup \ldots \cup \operatorname{Co}\left(D_{m}\right)\right)
$$


see Figure 8 below.
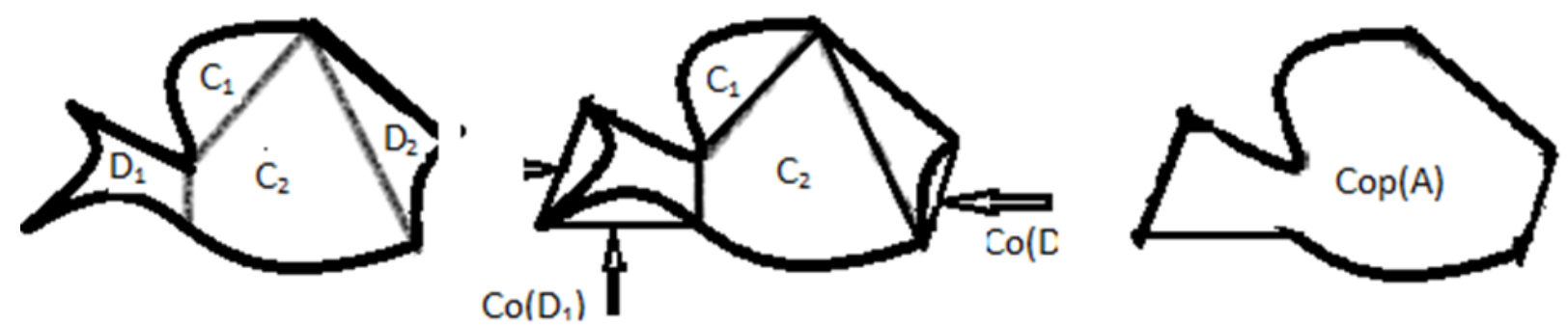

Figure (8): The subset A partitioned $A=(C 1 \cup C 2) \cup(D 1 \cup D 2)$ in the left, and the convexification of D1, D2 in the middle, and $\operatorname{Cop}(A)=(C 1 \cup C 2) \cup(\operatorname{co}(D 1) \cup \operatorname{co}(D 2))$ in the right.

Proposition 4.1. Let $\mathrm{E}$ a Saidou Space and $\mathrm{A}$ is a compact subset in $\mathrm{E}$.

Then, $\mathrm{A} \subset \operatorname{cop}(A) \subset \mathrm{Co}(\mathrm{A})$. see Figure 9 below.
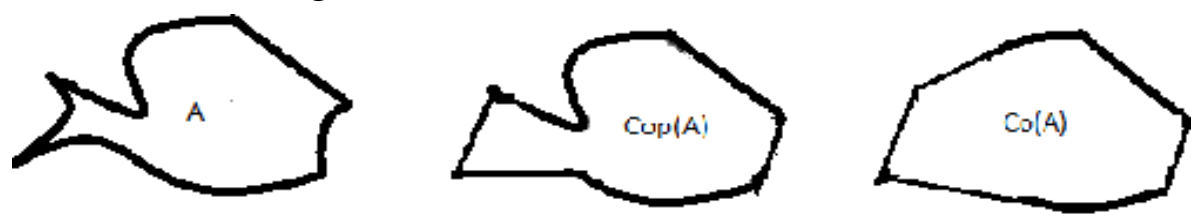

Figure(9): The subset $A$ in the left, its $\operatorname{Cop}(A)$ in the middle and $\operatorname{Co}(A)$ in the right.

We remark that $\operatorname{Cop}(\mathrm{A})$ in the middle is smaller than $\operatorname{Co}(\mathrm{A})$ in the left.

Proof 4.2 It clear that $D_{i} \subset \operatorname{Co}\left(D_{i}\right)$ for all $i$, then $U D_{i} \subset U \operatorname{Co}\left(D_{i}\right)$.

Therfore,

Hence

$$
A=\left(U C_{i}\right) \cup\left(U D_{i}\right) \subset\left(U C_{i}\right) \cup\left(U \operatorname{Co}\left(D_{i}\right)\right)=\operatorname{Cop}(A) .
$$

$$
A \subset \operatorname{Cop}(A) \text {. }
$$

Let $x \in \operatorname{Cop}(A)$, if $x$ is in $U\left(C_{i}\right)$ then there exists $i_{0}$ such that $x$ is in $C_{i} 0$ which is a subset of $\operatorname{Co}(A)$. If $x$ is in $U\left(\operatorname{Co}\left(D_{i}\right)\right)$, then there exists $i_{0}$ such that $x$ is in $\operatorname{Co}\left(D_{i 0}\right)$. Then, $x$ $=\sum \alpha_{\mathrm{i}} \mathrm{x}_{\mathrm{i}}, \sum \alpha_{\mathrm{i}}=1$, with $\mathrm{x}_{\mathrm{i}} \in \mathrm{A}$, which means that $\mathrm{x}$ is in $\operatorname{Co}(\mathrm{A})$.

Remark 4.3 We have denote that the convex envelop by part is not always convex. In case in $\mathrm{A}$ is a cartesian set it is easy to verify that $\operatorname{Cop}(\mathrm{A})=\mathrm{A}$, because we can partition $\mathrm{A}$ in convex parts see Figure 10 below.
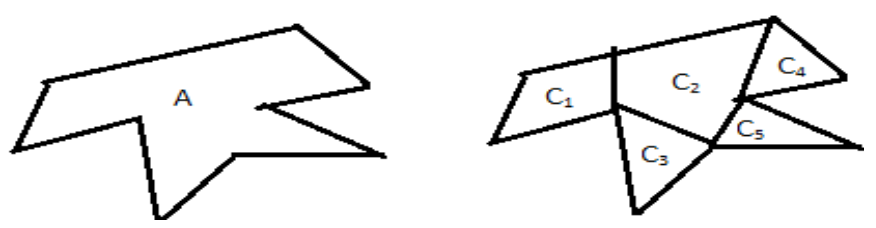

Figure(10): The subset $A$ in the left, its $\operatorname{Cop}(A)$ in the middle and $\operatorname{Co}(A)$ in the right. 
[7] collier [8].

Proposition 4.4. Let $\mathrm{E}$ a Banach space and $\mathrm{A}$ is a compact subset in $\mathrm{E}$. Then, $\mathrm{A}=\mathrm{Co}(\exp (\mathrm{A}))$. We refer to the Bourgin and Collier result, see [5], [4], [14].

Proposition 4.5. Let $G$ a finite subset of exposed point of $A$. then $\operatorname{Co}(G)$ is a cartesian set see Figure11 below.
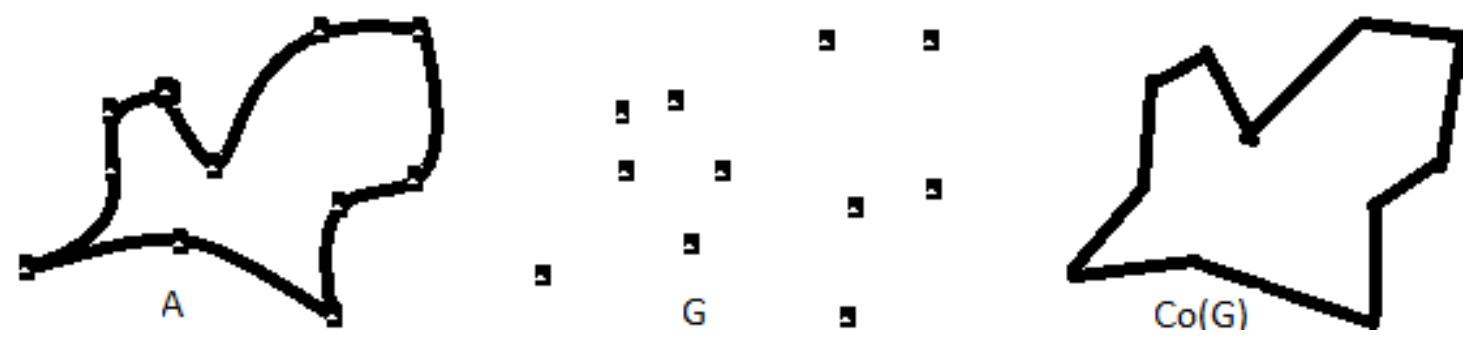

Figure(11): The subset $A$ with some exposed points, $G$ in the middle and $\operatorname{Co}(G)$ in the right.

Now, with all this preliminaries, we will be able to give the Algorithm of cartesianization of a compact subset A that is the aim of this work.

\section{Cartesianization Algorithm}

As we signaled, our objective is to construct an approximate cartesian set of a region A in $\mathrm{E}$ see Figure 1 above. The approximation will depends in the number of $N$ exposed points chosen from $\exp (A)$. Then we will call this method the $\mathrm{N}$-cartesianization of $A$. To say cartesianization in a such $\mathrm{E}$ is the equivalent to the polygonization in $R^{2} \operatorname{Ref}$ [12] [13], but these techniques are not the same.

Step I. Let $A$ a compact subset in E not necessarily convex. We partition $A$ to a finite compact convex parts and to a compact non convex parts. See Figure 7 above.

$$
A=\left(C_{1} \cup C_{2} \cup \ldots \cup C_{n}\right) \cup\left(D_{1} \cup \ldots \cup D_{m}\right)
$$

Step II. We construct the $\operatorname{Cop}(A)$ using the convexification by part as explained above see Figure 8 . Then $A$ is partionned in a finite union of compact convex parts $C_{i}$.

$$
A=\left(C_{1} \cup C_{2} \cup \ldots \cup C_{N}\right), N=m+n
$$

Since $C_{i}$, according to the proposition4.4 above, we have $C_{i}=\operatorname{co}\left(\exp \left(C_{i}\right)\right)$, for all $\left.i \in 1, . . N\right\}$. Let $G_{i}$ the subset of a finite exposed points of $C_{i}$.

Step III. We consider $\left.G=\cup G_{i}, i \in 1, \ldots N\right\}$ see Figure 12 below.
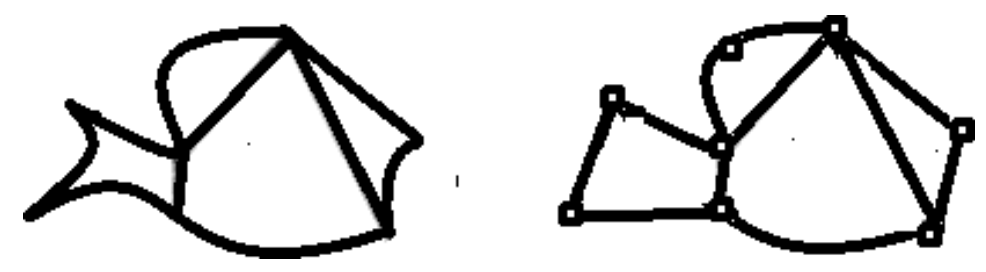

Figure(12): The subset $A$ partitioned in the right and each $C_{i}$ with $G_{i}$ in the right. 
Then, we construct the set.

$$
\operatorname{Car}_{N}(A)=\operatorname{Co}(G)
$$

\section{Conclusion:}

The set $\operatorname{Car}_{N}(A)$ is the N-profile cartesianized of A. See Figure 13 below.
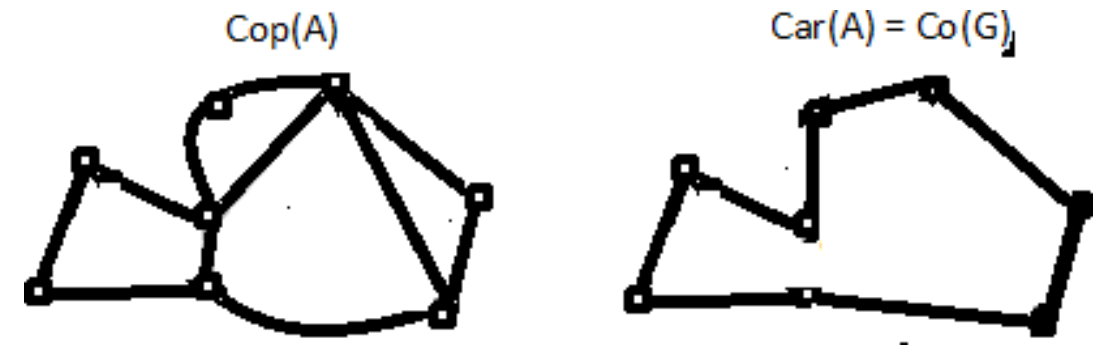

Figure(13): The subset $A$ with some exposed points, $\operatorname{Car}_{N}(A)$ in the right.

End of the Algorithm.

Remark 5.1 We remark that $\operatorname{Car}_{\mathrm{N}}(\mathrm{A})$ is an approximation of $\mathrm{A}$. Intuitively, as $\mathrm{N}$ increases so $\operatorname{much} \operatorname{Car}_{N}(\mathrm{~A})$ becomes closer to A. see Figure 14 below. And more than the boundary of A is round more than we need more exposed points to approximate as much as possible A.
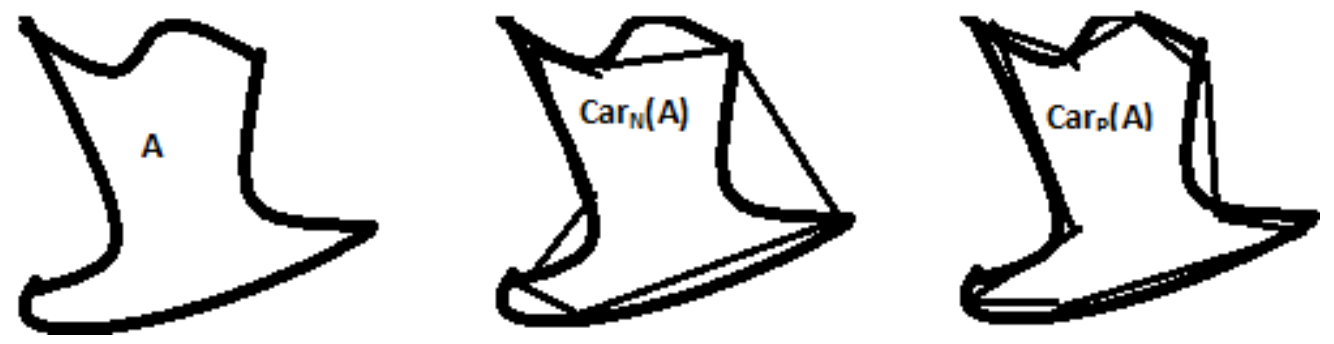

Figure (14): The subset $A$ in the left, its Cartesianization $\operatorname{Car}_{N}(A)$ with $N$ points in the middle, and its cartesianization $\operatorname{Car}_{P}(A)$ in with $p>N$ in the right.

In the next paper we will explain this method in the $\mathrm{R}^{2}$ set and give a cartesianization measure as a function of $\mathrm{N}$, the number of exposed points. In $\mathrm{R}^{2}$ the work has already been done in the form of polygonization Ref

[12] [13] [6] [7]. But our method will be based on the surface difference between A and Car(A) as a measure of Cartesianization. see Figure 15 below.
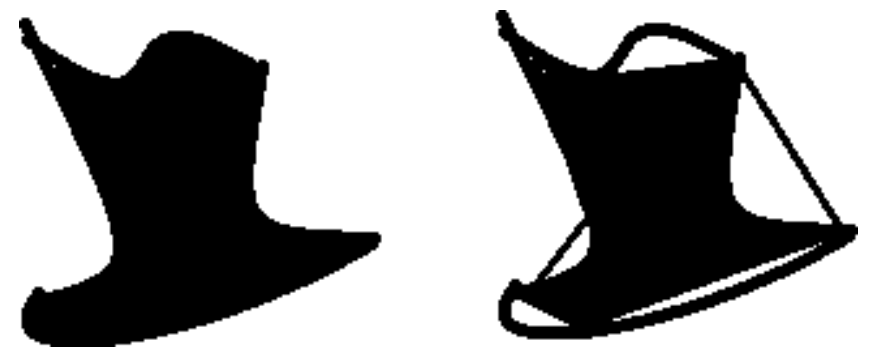

Figure (15): In the left the white surface is the surface difference between A and Car(A) which is a measure of cartesianization. 
In $\mathrm{R}^{2}$ and $\mathrm{R}^{3}$, we will digitize our Algorithm using Matlab or Silab as tool to program the technique explained above.

\section{References:}

[1] E. Bishop, R. R. Phelps, The support functionals of a convex set, Proc. Symp. in Pure Math, 7(1963), 27-35, https://doi.org/10.1090/pspum/007/0154092

[2] M. Brion, Points entiers dans les polytopes convexes, Seminaire Bour- baki, Vol. 1993/94, Exp. No. 780, Asterisque 227 (1995), 145-169.

[3] B. Cascales \& J. Orihuela, On Compactness in Locally Convex Spaces, Math. Z. 195, (1987)365381.

[4] J. B. Collier (1976), The dual of a space with the Radon-Nikodym prop- erty. Pacific J. Math, 64(1), 103-106, https://doi.org/10.2140/pjm.1976.64.103

[5] R.E. Huff \& P.D. Morris, Geometric characterizations of Radon- Nikodym proprety. In : Notices of the American Mathematical Society. 201 Charles ST, Providence, RI 02940-2213: Amer Mathematical Soc, (1975), p. A15-A15.

[6] N. Guyen, T.P., I. Debled-Rennesson, On the local properties of digital curves, International Journal of Shape Modeling 14(2)(2008), 105-125, https://doi.org/10.1142/s0218654308001105

[7] V. L. Klee, Polyhedral sections of convex bodies, Actu Math, 103(3-4)(1960), 243-267, https://doi.org/10.1007/bf02546358

[8] M. Krein \& D. Milman, On extreme points of regular convex sets, Studia Mathematica 9(1)(1940) 133-138, https://doi.org/10.4064/sm-9-1-133-138

[9] J. Lindenstraquss, Weakly compact sets-their topological properties and the Banach spaces they generate, Symp. on Infinite Dimensional Topology, Ann. of Math. Stud. 69, Princeton Univ. Press, (1972), 235-273, https://doi.org/10.1515/9781400881406-021

[10] H. B. Maynard, A geometric characterization of Banach spaces having the Radon-Nikodym property, Trans. Amer. Math. Sot. 185(1973), 493- 500.

[11] D.P. Milman, Characteristic of extremal functions, Dokl. Akad, Nank SSSR 57 (1947) I 119-122 (in Rus J. W. Roberts, A compact convex set with no extreme points, Studia Math. 60 (1977), no. 3, 255-266.

[12] T.P. Nguyen \& I. Debled-Rennesson, Curvature estimation in noisy curves. In: CAIP. Volume 4673 of LNCS. (2007) 474- 481, https://doi.org/10.1007/978-3-540-74272-2_59

[13] T.P. Nguyen. \& I. Debled-Rennesson, Segmentation en arcs discrets en temps linéaire. In: RFIA. (2010)

[14] R. R. Phelps, Dentability and Extreme Points in Banach Spaces, Jour- Nal of Functional Analysis, 17(1)(1974), 78-90, https://doi.org/10.1016/0022-1236(74)90005-6

[15] N. Saidou, Mathematical introduction to Cartesian geometry in complex shapes. Accepted in Mathematics and computer sciences, to appear.

[16] H. WE, The elementary theory of convex polyhedra, Annals of Mathe- matical Studies, 24(1950), 3-18. 